\title{
Earthquake Analysis, of RC Structure using Different Codes and Different Countries
}

\author{
Gaurav Charavande, Savita Maru
}

\begin{abstract}
This paper presents a seismic behavior of various structures using different codal provision as given Indian code, American code, \&Newzealand code for earthquake analysis. This study is carried out on residential building of $G+5, G+11, G+21$ of Special RC structure. Modeling of the structure is done as per ETAB software. Time period of the structure in both the direction is taken from the software as per the three standard (9 model are made 3 model for each code). A comparative analysis is performed in terms of base shear, deflection limit, stores drift at linearly static and response spectrum.
\end{abstract}

Key Words: Base Shear, Displacement, Seismic Analysis , Storey Drift

\section{INTRODUCTION}

Structural design is a workmanship and study of understanding the conduct of basic part of oppressed powers and loads and structuring them with economy and style to give a protected, useful and tough. Structural design of structures of any nation depends on specific codes of practice which give the fundamental information and norms in breaking down and planning the structure from quality perspective and affordable perspective. These codes are completed by profoundly experienced basic architects, academicians and other prominent colleagues of separate regions. This paper concerned the comparison of nominal loads, load combination, load factor, design parameters like beam, column and beam and their suitability from various building codes. The utilization of various plan strategies and codes give various outcomes in basic investigation and structure that prompts changeability in conduct, expenses and strength of structures. Such examination gives heaps of data identified with basic structure that at what degree one nation"s code vary from another nation"s code as far as level of exactness, security, multifaceted nature and subtleties are considered. Thus, it is the obligation of basic designer to give precise measures that lead to ideal execution and economy by with respect to the most proper structure technique. Such inventive capacity and creative mind is completely founded on understanding of auxiliary architects. The auxiliary plan procedure includes basic arranging, activity of powers and loads, part investigation, part structure, correlation among various structures codes and their itemizing.

Revised Manuscript Received on December 30, 2019.

* Correspondence Author

Gaurav Charavande, Post-Graduation Student, Ujjain Engineering College, Ujjain, Rgpv University, Bhopal M.P, India E-Mail: Gauravcharavande27@Gmail.Com

Savita Maru, Professor, Department of Civil Engineering, Ujjain Engineering College, Ujjain, Rgpv University, Bhopal M.P, India Ujjain,456010, Madhya Pradesh , India E-mail: savitamaru@yahoo.com

(c) The Authors. Published by Blue Eyes Intelligence Engineering and Sciences Publication (BEIESP). This is an open access article under the CC BY-NC-ND license (http://creativecommons.org/licenses/by-nc-nd/4.0/)
It is checked on that those nations where more than one code is received for basic plan so it is useful in establishing what code has higher factor of security and level of precision than another.

\subsection{Objective of the project:}

The main objective of this project is to bring out the most causative factors that cause poor performance throughout the earthquake and build recommendations that ought to be taken under consideration in coming up with the multistoreyed concrete buildings therefore on bring home the bacon their adequate safe behaviour. Indian common place Code IS:1893 was appropriately update in 2016 therefore on address the assorted style problems brought call at the earthquake behaviour of the RC Buildings. The chosen standards are Indian Standard Code (IS:1893), American code (ASCE-7-2002), \& Newzealand code(1170.2004) . A comparative analysis was performed in terms of Base shear, Displacement, for different codes.

\subsection{Methodology:}

The methodology worked out to achieve the mentioned objectives is as follows:

1. Modelling of the selected building in Etab Software.

2. Three models as per the codes i.e. Indian code, American code , Newzealand code specification were made.

3. Applied manually calculated Lateral seismic forces and load combinations as per IS 1893-2016, American code (ASCE-7-2002), \& Newzealand code(1170.2004)

4. Analyzed the models and graphical and tabular representation of the data is presented.

\section{MODELLING}

A multi-storey building of $\mathrm{G}+5, \mathrm{G}+11$, and $\mathrm{G}+21$, Special RC moment-resting frame (SMRF) is taken for study. The typical storey height is $3.2 \mathrm{~m}$ for all storey.

The three codal provisions as mentioned above. A Linear Static analysis is done using Etab Software. The model is studied for all three code for severe parameter with medium soil condition.

\subsection{Plan and Specification of the Building}

:- MODEL: Plan of the building 
Earthquake Analysis, of RC Structure using Different Codes and Different Countries

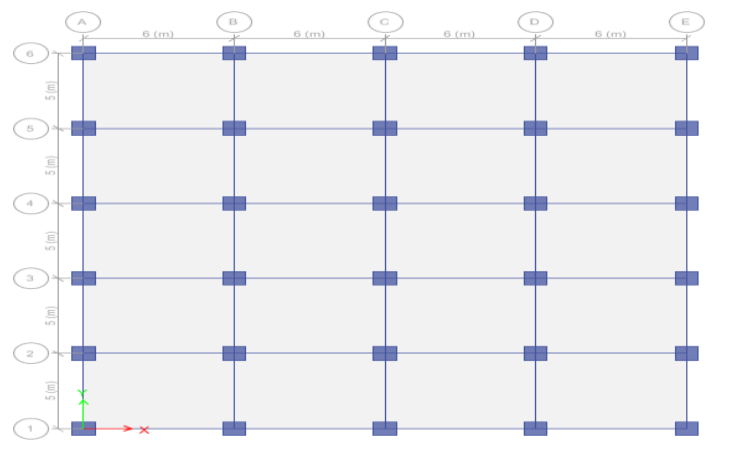

Fig (1). Plan of Building

Table -2.2 : Specification of the Building

\begin{tabular}{|c|c|c|}
\hline SR NO. & Parameters & Dimension/Type \\
\hline 1 & Plan Dimension & $25 \mathrm{~m} \times 24 \mathrm{~m}$ \\
\hline 2 & No. Of Stories & G+5, G+11, G+21 \\
\hline 3 & $\begin{array}{c}\text { Height of Each } \\
\text { Storey }\end{array}$ & $3.2 \mathrm{~m}$ \\
\hline 4 & Grade of Concrete & M30 \\
\hline 5 & Frame Type & SMRF \\
\hline 6 & Soil Type & Medium Soil \\
\hline 7 & Column Size & $450 \mathrm{~mm}, 600 \mathrm{~mm}, 900 \mathrm{~mm}$ \\
\hline 8 & Beam Size & $250 \mathrm{~mm}, 450 \mathrm{~mm}, 500 \mathrm{~mm}$ \\
\hline 9 & Slab Thickness & $25 \mathrm{~mm}$ \\
\hline 10 & $\begin{array}{c}\text { Unit Weight of } \\
\text { Concrete }\end{array}$ \\
\hline
\end{tabular}

III. ANALYSIS AND RESULTS

Result obtained for Base Shear

Case (i). Result for Base Shear in X-Direction

Max base shear

\begin{tabular}{|c|c|c|c|}
\hline STORY & $\begin{array}{c}\text { INDIA } \\
\text { X-Dir mm }\end{array}$ & $\begin{array}{c}\text { ACI } \\
\text { X-Dir mm }\end{array}$ & $\begin{array}{c}\text { NZS } \\
\text { X-Dir mm }\end{array}$ \\
\hline 5 & 1956.936 & 556.532 & 2419.568 \\
\hline $\mathrm{G}+11$ & 2449.362 & 3078.322 & 9697.909 \\
\hline $\mathrm{G}+21$ & 3339.521 & 5470.615 & 9697.909 \\
\hline
\end{tabular}

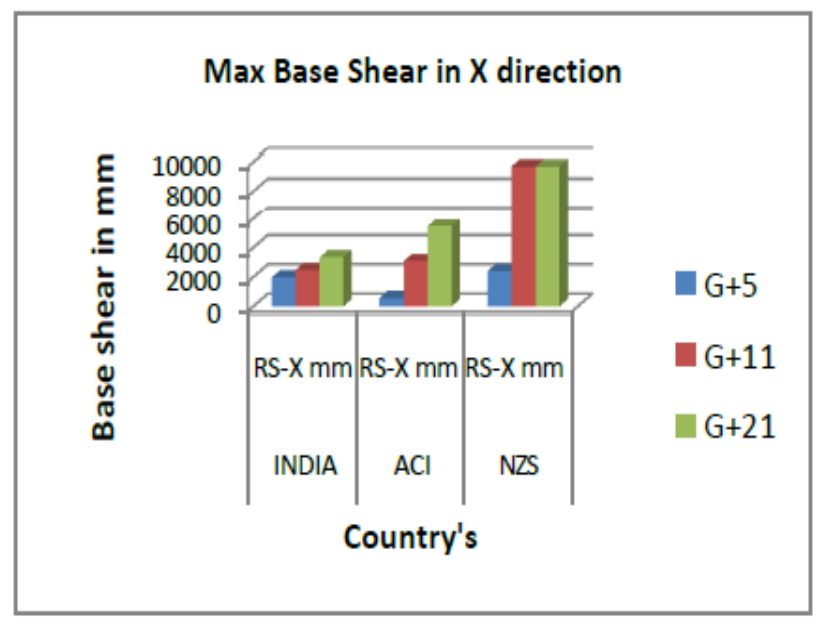

Case (ii). Result for Base Shear in Y- Direction

Max base shear

\begin{tabular}{|c|c|c|c|}
\hline STORY & $\begin{array}{c}\text { INDIA } \\
\text { Y-Dir mm }\end{array}$ & $\begin{array}{c}\text { ACI } \\
\text { Y-Dir mm }\end{array}$ & $\begin{array}{c}\text { NZS } \\
\text { Y-Dir mm }\end{array}$ \\
\hline G+5 & 1956.936 & 556.532 & 2536.676 \\
\hline G+11 & 2449.362 & 3078.322 & 9697.909 \\
\hline G+21 & 3339.521 & 5470.615 & 9697.909 \\
\hline
\end{tabular}

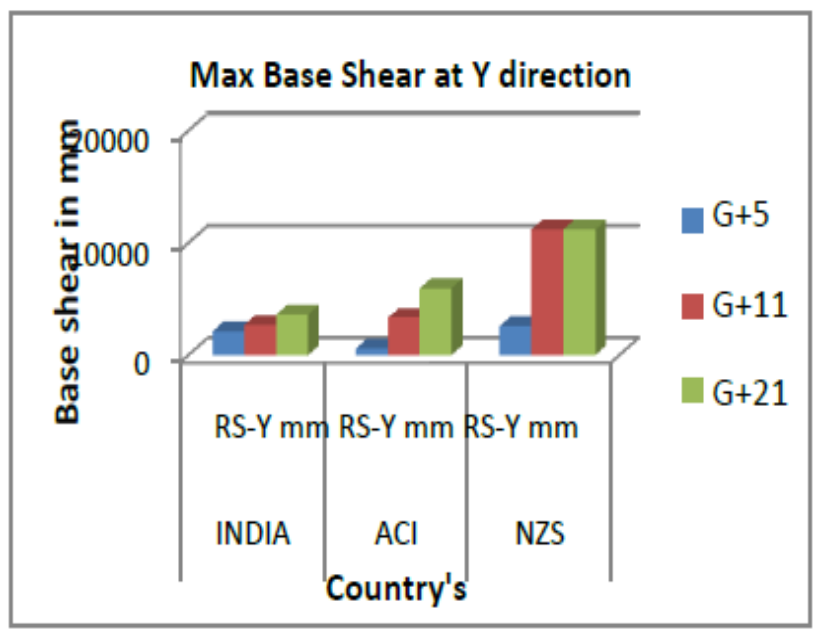

Result obtained for Displacement.

Result for $\mathbf{G + 5}$

Max story displacement

\begin{tabular}{|l|l|l|l|}
\hline STORY & $\begin{array}{l}\text { INDIA } \\
\text { mm }\end{array}$ & $\begin{array}{l}\text { ACI } \\
\text { mm }\end{array}$ & $\begin{array}{l}\text { NZS } \\
\text { mm }\end{array}$ \\
\hline Story6 & 36.7 & 43.18 & 41.4 \\
\hline Story5 & 33.2 & 38.1 & 37.7 \\
\hline Story4 & 27.6 & 33.02 & 31.8 \\
\hline Story3 & 20.4 & 25.4 & 24.0 \\
\hline Story2 & 12.5 & 15.24 & 14.9 \\
\hline Story1 & 4.7 & 5.08 & 5.7 \\
\hline Base & 0 & 0 & 0 \\
\hline
\end{tabular}

Published By: 


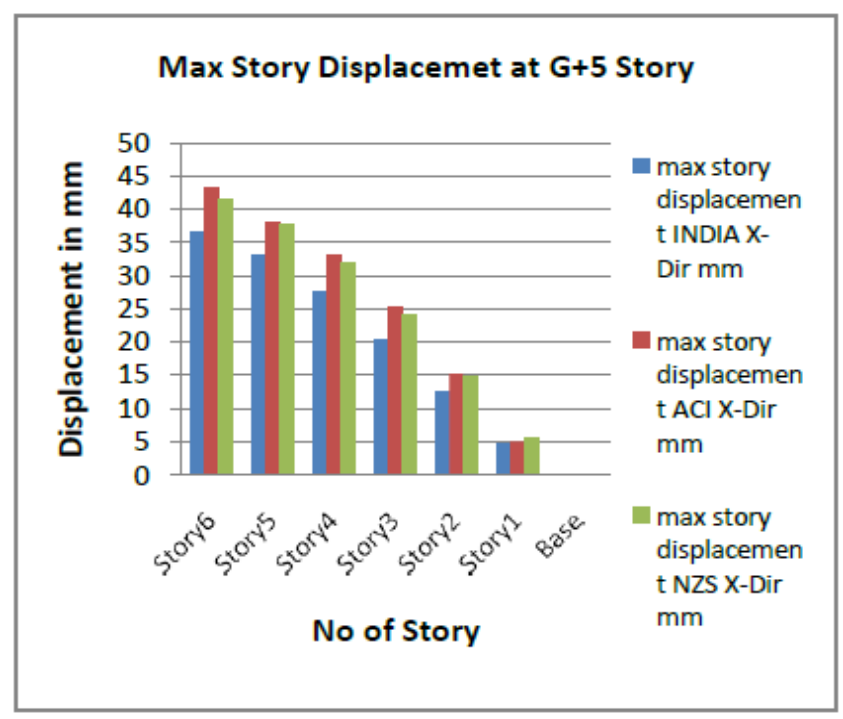

Result for $\mathbf{G + 1 1}$

Max story displacement

\begin{tabular}{|c|c|c|c|}
\hline STORY & $\begin{array}{c}\text { INDIA } \\
\mathbf{~ m m}\end{array}$ & $\begin{array}{c}\text { ACI } \\
\mathbf{~ m m}\end{array}$ & $\begin{array}{c}\text { NZS } \\
\mathbf{~ m m}\end{array}$ \\
\hline Story12 & 76.8 & 90.4 & 129.3 \\
\hline Story11 & 74.2 & 87.6 & 124.9 \\
\hline Story10 & 70.3 & 83.5 & 118.7 \\
\hline Story9 & 65.3 & 77.9 & 110.7 \\
\hline Story8 & 59.1 & 71 & 101 \\
\hline Story7 & 52 & 62.9 & 89.7 \\
\hline Story6 & 44.2 & 53.9 & 77 \\
\hline Story5 & 35.9 & 44.1 & 63.3 \\
\hline Story4 & 27.4 & 33.8 & 48.7 \\
\hline Story3 & 18.7 & 23.3 & 33.7 \\
\hline Story2 & 10.5 & 13 & 19 \\
\hline Story1 & 3.5 & 4.3 & 6.3 \\
\hline Base & 0 & 0 & 0 \\
\hline
\end{tabular}

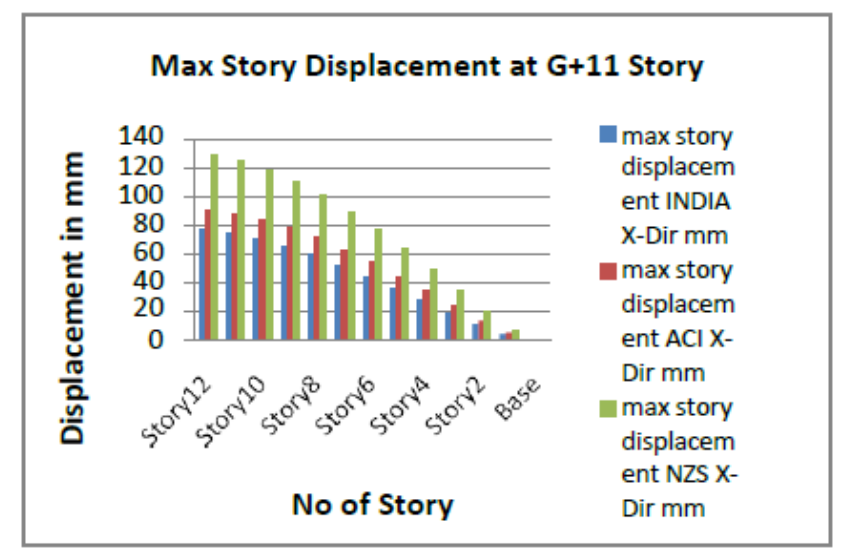

Result for $\mathrm{G}+21$

Max story displacement

\begin{tabular}{|c|c|c|c|}
\hline STORY & $\begin{array}{c}\text { INDIA } \\
\mathbf{m m}\end{array}$ & $\begin{array}{c}\text { ACI } \\
\mathbf{m m}\end{array}$ & $\begin{array}{c}\text { NZS } \\
\mathbf{m m}\end{array}$ \\
\hline Story22 & 118.8 & 190.6 & 315.3 \\
\hline Story21 & 116.8 & 187.4 & 309.6 \\
\hline Story20 & 114.3 & 183.6 & 303.0 \\
\hline Story19 & 111.3 & 178.8 & 295.1 \\
\hline
\end{tabular}

Retrieval Number: B2915129219/2019@BEIESP

DOI: 10.35940/ijeat.B2915.129219

Journal Website: www.ijeat.org

\begin{tabular}{|l|c|c|c|}
\hline Story18 & 107.6 & 173.2 & 285.9 \\
\hline Story17 & 103.4 & 166.6 & 275.4 \\
\hline Story16 & 98.7 & 159.1 & 263.6 \\
\hline Story15 & 93.4 & 150.8 & 250.6 \\
\hline Story14 & 87.7 & 141.8 & 236.4 \\
\hline Story13 & 81.7 & 132.2 & 221.2 \\
\hline Story12 & 75.2 & 121.9 & 204.9 \\
\hline Story11 & 68.6 & 111.2 & 187.8 \\
\hline Story10 & 61.6 & 100.1 & 169.8 \\
\hline
\end{tabular}

\begin{tabular}{|c|c|c|c|}
\hline Story9 & 54.5 & 88.7 & 151.1 \\
\hline Story8 & 47.3 & 77.0 & 131.9 \\
\hline Story7 & 40.0 & 65.2 & 112.2 \\
\hline Story6 & 32.7 & 53.4 & $\mathbf{r 9 2 . 2}$ \\
\hline Story5 & 25.5 & 41.6 & 72.2 \\
\hline Story4 & 18.5 & 30.2 & 52.6 \\
\hline Story3 & 12.0 & 19.5 & 34.1 \\
\hline Story2 & 6.2 & 10.1 & 17.7 \\
\hline Story1 & 1.8 & 3.0 & 5.3 \\
\hline Base & 0 & 0 & 0 \\
\hline
\end{tabular}

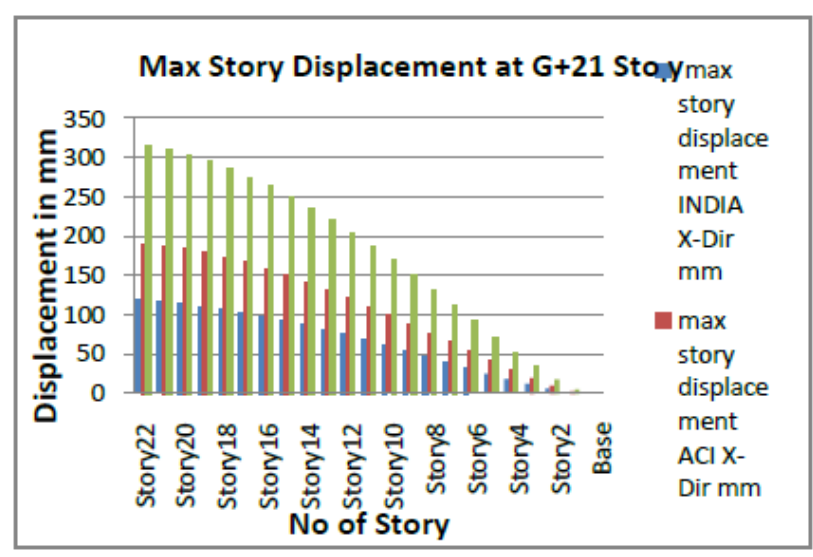

\section{Result obtained for Storey Drift}

Result for $\mathbf{G}+5$

Max story drift

\begin{tabular}{|c|c|c|c|}
\hline STORY & INDIA & ACI & NZS \\
\hline Story6 & 0.001097 & 0.001143 & 0.001167 \\
\hline Story5 & 0.001753 & 0.001913 & 0.001854 \\
\hline Story4 & 0.002231 & 0.00257 & 0.002429 \\
\hline Story3 & 0.002483 & 0.003011 & 0.002824 \\
\hline Story2 & 0.002433 & 0.003074 & 0.002884 \\
\hline Story1 & 0.00147 & 0.001904 & 0.001791 \\
\hline Base & 0 & 0 & 0 \\
\hline
\end{tabular}


Earthquake Analysis, of RC Structure using Different Codes and Different Countries

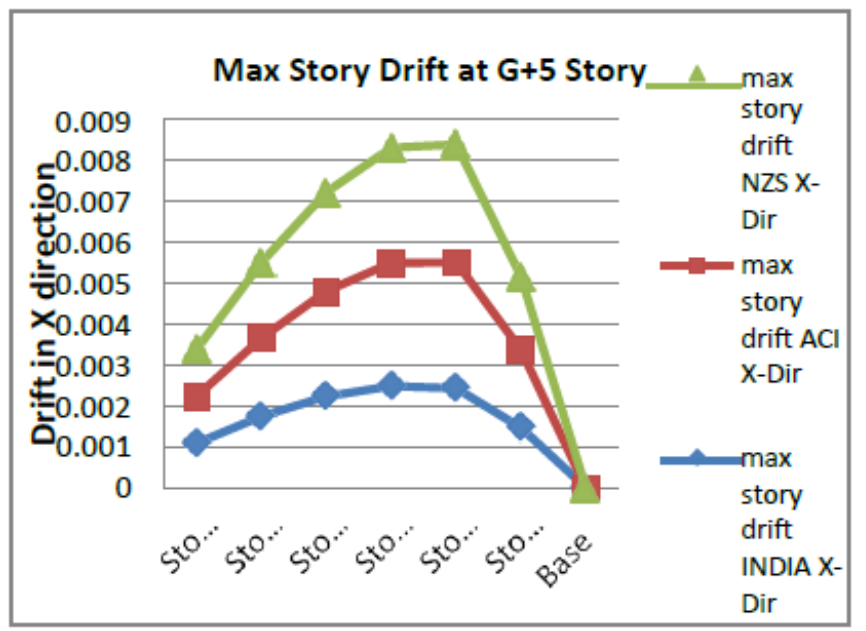

Result for G+11

Max story drift

\begin{tabular}{|c|c|c|c|}
\hline STORY & INDIA & ACI & NZS \\
\hline Story12 & 0.000824 & 0.00088 & 0.001381 \\
\hline Story11 & 0.001201 & 0.001295 & 0.00193 \\
\hline Story10 & 0.00159 & 0.001744 & 0.002501 \\
\hline Story9 & 0.001933 & 0.00216 & 0.003039 \\
\hline Story8 & 0.002214 & 0.002522 & 0.003525 \\
\hline Story7 & 0.00243 & 0.002823 & 0.003947 \\
\hline Story6 & 0.002585 & 0.003058 & 0.004298 \\
\hline Story5 & 0.002678 & 0.003222 & 0.004562 \\
\hline Story4 & 0.002695 & 0.00329 & 0.004698 \\
\hline Story3 & 0.002586 & 0.003192 & 0.004597 \\
\hline Story2 & 0.002195 & 0.002731 & 0.003961 \\
\hline Story1 & 0.00108 & 0.00135 & 0.001968 \\
\hline Base & 0 & 0 & 0 \\
\hline
\end{tabular}

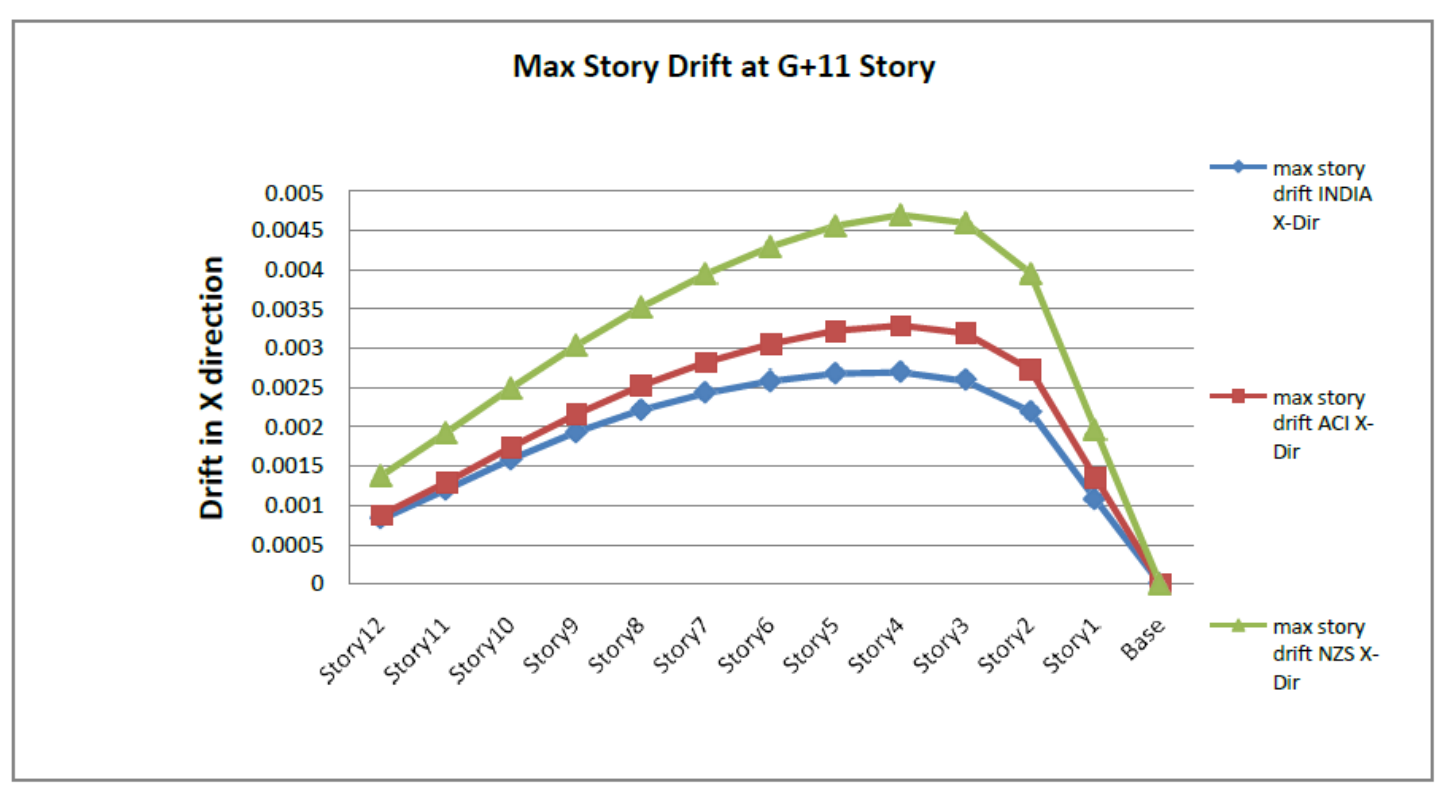

Result for G+21

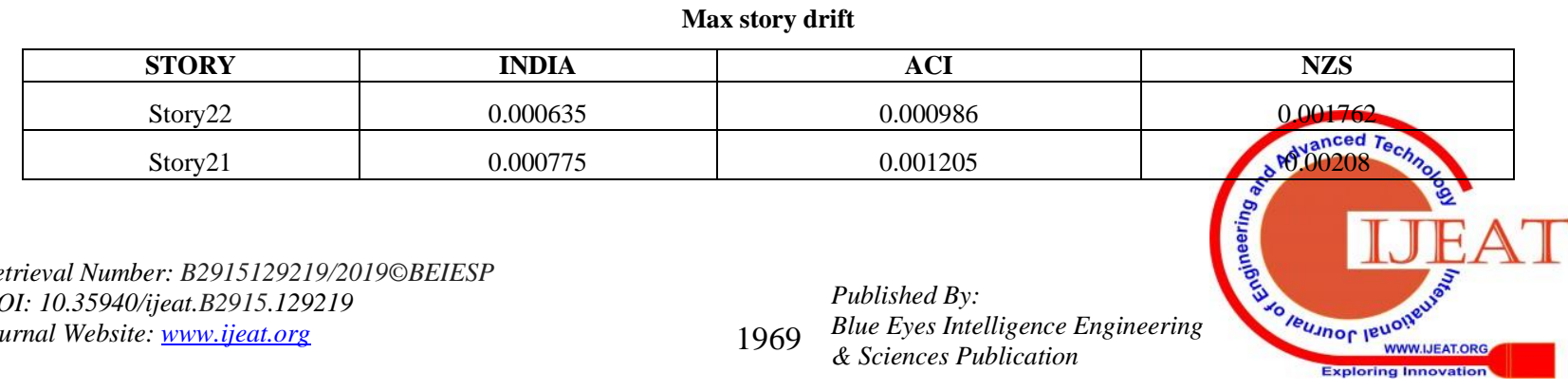




\begin{tabular}{|c|c|c|c|}
\hline Story20 & 0.00095 & 0.00148 & 0.002463 \\
\hline Story19 & 0.001135 & 0.001773 & 0.002871 \\
\hline Story18 & 0.001316 & 0.002061 & 0.003281 \\
\hline Story17 & 0.001486 & 0.002335 & 0.003682 \\
\hline Story16 & 0.00164 & 0.002586 & 0.004065 \\
\hline Story15 & 0.001778 & 0.002814 & 0.004427 \\
\hline Story14 & 0.001899 & 0.003016 & 0.004765 \\
\hline Story13 & 0.002003 & 0.003193 & 0.005076 \\
\hline Story12 & 0.002091 & 0.003344 & 0.00536 \\
\hline Story11 & 0.002162 & 0.00347 & 0.005615 \\
\hline Story10 & 0.002218 & 0.003572 & 0.005836 \\
\hline
\end{tabular}

\begin{tabular}{|c|c|c|c|}
\hline Story9 & 0.002258 & 0.003647 & 0.006021 \\
\hline Story8 & 0.00228 & 0.003693 & 0.006161 \\
\hline Story7 & 0.002282 & 0.003704 & 0.006243 \\
\hline Story6 & 0.002254 & 0.003667 & 0.006242 \\
\hline Story5 & 0.002185 & 0.003561 & 0.006115 \\
\hline Story4 & 0.002049 & 0.003344 & 0.005786 \\
\hline Story3 & 0.0018 & 0.002942 & 0.005122 \\
\hline Story2 & 0.001358 & 0.00222 & 0.003886 \\
\hline Story1 & 0.000576 & 0.000943 & 0.001656 \\
\hline Base & 0 & 0 & 0 \\
\hline
\end{tabular}

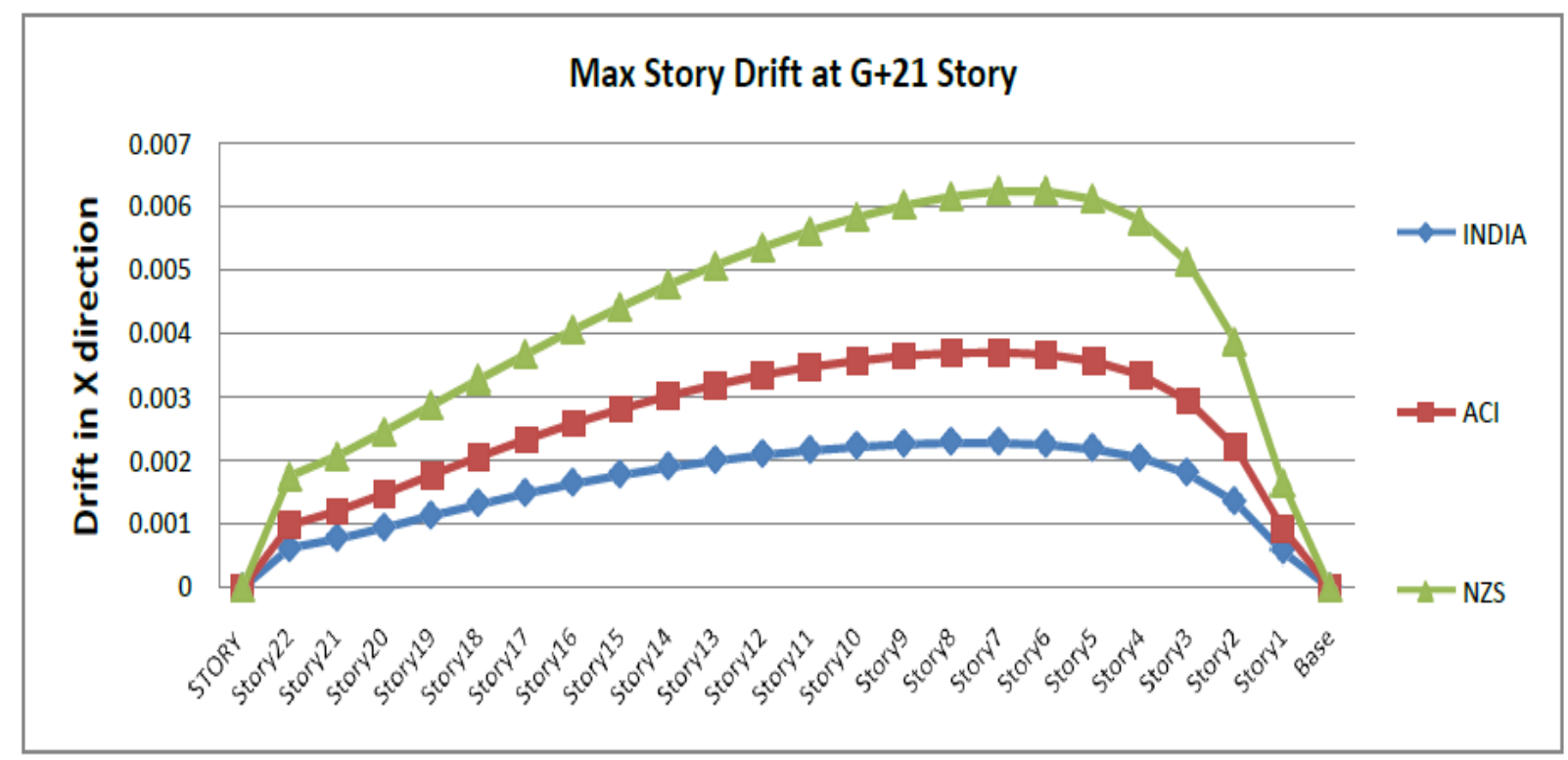

\section{CONCLUSION}

\section{Conclusions for Base Shear For $G+5$}

When base shear was Calculated in X-Y direction, American code showed better results than Indian code, Newzealand code.

When Newzealand and Indian code were compared, Indian

\section{For G+11}

When base shear was Calculated in $\mathrm{X}-\mathrm{Y}$ direction, Indian code showed better results than American code, Newzealand code.

When Newzealand and American code were compared , code gave low base shear.

American code gave low base shear.

\section{For G+21}

When base shear was Calculated in $\mathrm{X}-\mathrm{Y}$ direction, Indian code showed better results than American code, Newzealand code.

When Newzealand and American code were compared , American code gave low base shear.

\section{Conclusions for Story Drift For G+5}

When Story Drift was Calculated, Indian code showed better results than American code, Newzealand code. American code gave Max storey drift which is unaccettable.

Published By:

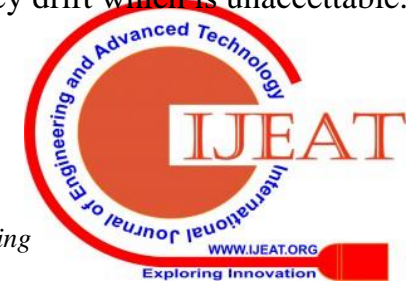




\section{Earthquake Analysis, of RC Structure using Different Codes and Different Countries}

\section{For G+11}

When Story Drift was Calculated, Indian code showed better results than American code, Newzealand code. Newzealand code gave Max storey drift which is unaccettable.

\section{For $\mathbf{G}+21$}

When Story Drift was Calculated, Indian code showed better results than American code, Newzealand code.. Newzealand code gave Max storey drift which is unaccettable.

\section{Conclusions for Displacement}

For G+5 When Story Displacement was Calculated , Indian code showed better results than American code, Newzealand code.

Newzealand code gave Max storey drift which is unaccettable at story 6

\section{For $\mathbf{G}+11$}

When Story Displacement was Calculated, Indian code showed better results than American code, Newzealand code. Newzealand code gave Max storey drift which is unaccettable at storey 12.

\section{For $\mathbf{G}+21$}

When Story Displacement was Calculated, Indian code showed better results than American code, Newzealand code. Newzealand code gave Max storey drift which is unaccettable at storey 22.

\section{REFERENCES}

1. IbnuRusyd, Muksin Umar and LubnaAlam (2018), "A GIS-Based earthquake modeleee. A case study at university of the Philippines Los Banes, Philippines journal of science, vol.147, no.2,pp.301-316,2018, ISSN:0031-7683.

2. SamreenBano (2018), "Comparative study of design of structural member of RC building on code for different countries ${ }^{\text {eee }}$. and Technology, vol.7, ISSN:2319-8753.

3. C.U.Mwoji and A.I.UGWU (2017), "Compare and Study of BS8110 and Eurocode 2 in structural design and analysisece. Nigerian Journal of Technology (NIJOTECH), vol.36, no.3, pp.758-766, 2017.

4. KamaldeepKaur and Jaspal Singh (2017), "Comparison of Seismic Behavior of RC structures using various codes ${ }^{\text {eeee }}$.International journal of Agriculture, Environment and Biotechnology, vol:10.5958/2230732x.2017

5. S.H.C.Santos (2017), "Comparative study of codes for seismic design of structures ${ }^{\text {eece }}$.VERSITA, vol.9-No.1-2013.

6. Pamela Jennifer, Jegidha. K., Sureshbabu, (2016) "Seismic Design of Multi-storied RC Building Using Various Codes" Internationa Journal of Research in Engineering and Technology, Volume:05 Issue: 02/ Feb-2016

7. Khan and Prasad (2016), “A comparative study of seismic behavior on multistoried RC buildings by the provision made in India and other International building codes. Int.J.Eng.Dev.Res., 4:1967-73.

8. Swajit Gaud (2016), "Comparative study on material used in various codes for design of RC and steel structure." The master builder, Research Gate.

9. Mourad M and Bakhoun (2015), "Comparison of action and resistances in different building codes. ${ }^{\text {cee }}$ Journal of advance research 2015/10.1016/j.jare.2015.11.001.

10. T.C. Nwofar (2015), "A comparative study of BS8110 and Eurocode2 standard for the design of a continuous reinforced concrete vol6, no5, pp.76-84, 2015, ISSN online: 0976-6316.

11. S. Karthiga (2015), "Design and comparison of a residential building (G+10) for seismic forces using the codes: IS1893, EUROCODE8, ASCE710 and BRITISH CODE. „Internal journal of research in engineering and technology, vol.4, no.6, 2015, ISSN online: 23191163.

12. RajmahendraManikaroSawant (2015), "Behavior of high strength fiber reinforced concrete under shear.”International journal of civil International Journal of Innovative Research in Science, Engineering beams.'International journal of civil engineering and technology,

engineering and technology, vol.6no.4pp.46-54,2015, ISSN online:0976-6316

13. Lakshmi K.O, Prof, Jayashree Ramanujan, Mrs. Bindu Sunil, Dr. Laju Kottallil, Prof. Mercy Joseph Poweth,(2014) "Effect of Shear wall location in buildings subjected to seismic loads" ISOI Journal of Engineering and Computer Science, Volume 1 Issue 1;2014, Page No. 07-17.

14. LabaniNandi ,PriyabrataGuha, (2014) "Design comparison of different structural element by using different international codes.'International journal of engineering research and technology (IJERT), vol.3, no3, 2014, ISSN: 2278-0181.

15. Ali.S.Alnuaimi (2013), "Design results of RC members subject to bending, shear and torsion using ACI 318:08 and BS 8110:97 buildings code, "Practice periodical on structural design and construction, vol.18,no.4,2013,ISSN:1084-0680.

16. SatyaPrakash Mishra (2012), "Comparison of IS, BS and ACI methods of concrete mix design and proposing function equation based design, "International journal of civil, structural, environmental and infrastructure engineering research and development (IJCSEIERD),vol.no.1,pp.20-56,2012,ISSN:2249-6866.

17. C.M Chan. M.F. Huang (2010), "Optical wind resistant performancebased design of tall buildings, "19 analysis and computation specialty conference@2010 ASCE.

18. Alice E. Diaz De Leon (2009), "National building code of India and the International building code: An Introduction", Indo-US forensic engineering workshop 2010.

19. Richard Fenwick, GreogoryMacral (2009), "Comparison of Newzealand standards used for seismic design of concrete

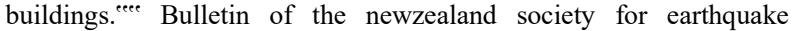
engineering, vol 42, no.3, 2009.

20. MarjanFaizain , Yuji Ishiyama (2004), "Comparison of seismic codes of 1981 JAPAN (BSLJ), 2000 USA (IBC) and 1999 IRAN (ICS) ${ }^{\text {ecee }} 13$ World Conference on Earthquake Engineering, Paper no.3168,2004.

21. Weizi Zhang and Bahram M. Sharooz (1999), "Comparison between ACI and AISC for concrete- filled tubular columns, ${ }^{\text {eee }}$ Journal of structural engineering, vol.125, no.11,1999,@ASCE, ISSN: 07339445.

22. Earthquake Response Spectra and Design Spectra-368961

23. ASCE/SEI 7-10. Minimum design loads for buildings and other structures. ASCE standard, American Society of Civil Engineering Institute.

24. Response- Spectrum-compatible ground motion processes-382576

25. AS/NZS 1170:2002. Structural design actions. Standards Australia/Standards New Zealand.

26. NZS 1170.5 Supp 1:2004. Structural Design Action Part 5: Earthquake actions- New Zealand-Commentary. Standards New Zealand.

27. Revised IS Code for Earthquake Resistant Design of Structures IS 1893 (Part 1) : 2002

28. Indian Standard Plain And Reinforced Concrete Code of practice IS 456:2000.

\section{AUTHORS PROFILE}

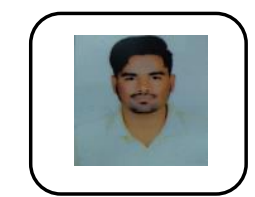

Gaurav Charavande, PG Scholar, Doing his Master of Engineering(ME) in Computer Aided Structural Design \& Drafting (CASDD) at Ujjain Engineering College,Ujjain.Teaching. Email id : gauravcharavande27@gmail.com

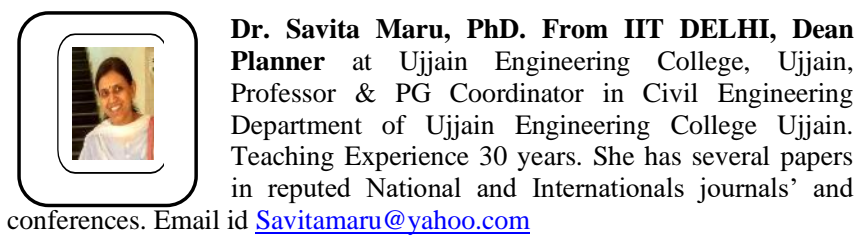

